Hier können Sie CME-Punkte sammeln a) für die Pflichtfortbildung aller Vertragsärzte und b) für freiwillige Fortbildungszertifikate, die viele Landesärztekammern anbieten.

Die Multiple-Choice-Fragen beziehen sich auf den vorangegangenen Fortbildungsbeitrag (Seiten 47-51). Die Antworten ergeben sich z.T. aus dem Text, z. T. beruhen sie auf medizinischem Basiswissen!

Wenn Sie 70\% der Fragen richtig beantworten, erhalten Sie 2, bei 100\% 3 CME-Punkte.

Einsendeschluss: 13. April 2008 (www.cme-punkt.de) bzw. 27. Dezember 2007 (Teilnahme per Brief).
Dr. H.J. K. Barwitz, Prof Dr. A. Berghaus, Prof Dr. Dr. h.c. Th. Brandt, Prof. Dr. W. G. Daniel, Prof. Dr. W. Eisenmenger, Prof. Dr. K. Friese, Prof. Dr. H. S. FüeßI, Prof. Dr. B. Göke, Prof. Dr. R. Gradinger, Prof. Dr. Dr. h.c. A. Hofstetter, Prof. Dr. H. Holzgreve, Prof. Dr. A. Imdahl, Prof. Dr. K. Krüger, Prof. Dr. H.-J. Möller, Prof. Dr. Dr. h.c. G. Plewig, Prof. Dr. D. Reinhardt, U. Weigeldt.

\title{
Fragen zum Thema „Symptom Husten“
}

\section{Welche Aussage trifft zu? Husten}

A spielt als Schutzreflex der oberen Atemwege eine untergeordnete Rolle.

B ist Teil des nasalen Clearancemechanismus.

C ist gemeinsames Symptom beinahe aller pulmonaler und einiger extrapulmonaler Erkrankungen.

D trägt im Gegensatz zum Niesen nicht zur Ausbreitung von Keimen bei.

$\mathrm{E}$ ist generell nicht behandlungsbedürftig.

- 2. Zu den insgesamt fünf Abschnitten des Hustenreflexbogens gehört/gehören

A der vagovasale Schenkel.

B der sympathische Schenkel.

C die lateralen Leitungsbahnen.

D Hustenrezeptoren.

E Beta-2-Rezeptoren.

\section{- 3. Husten kann ausgelöst werden durch}

A Lachen.

B Infekte der Atemwege.

C eine pleurale Reizung.

D kardiale Erkrankungen.

E Alle Aussagen sind richtig.

- 4. Welche Aussage zu Hustenmedikamenten trifft zu?

A Die Wirkung von Demulzentia hält in der Regel 24 Stunden an.

B Wenn inhalative Kortikosteroide helfen, ist Asthma unwahrscheinlich.

C Lokalanästhetika werden als Verneblertherapie bei persist. Husten empfohlen.

D Kodein ist ein Morphinanalogon und wirkt antitussiv.

E Zentralwirksame Antitussiva haben kein Suchtpotenzial.

\section{- 5. Welche Maßnahme/-n ist/sind hie erforderlich?}

A Hämoptysen und Husten: Abwarten und nach 14 Tagen kontrollieren.

B Dyspnoe und frühmorgendlicher Husten: u.a. Asthmaverdacht abklären.

C Plötzlicher Husten nach Nahrungsaufnahme: Keine Maßnahme, da es sich um einen harmlosen Effekt handelt, der häufig bei kalten Speisen auftritt.

D Husten nach Zigarettenrauchinhalation: Lungenfunktionstest obligat.

E Husten bei Inspiration: Bronchoskopie empfohlen.

- 6. Eine Erkrankung mit typischerweise akutem Husten ist

A Vocal Cord Dysfunction (VCD).

B Endokarditis.

C Tumoren im Bronchialbaum.

D Bronchitis bei Rauchern.

E Sinusitis.

\section{Welche Aussage zum akuten Husten trifft zu?}

A Vergiftungen können akuten Husten mit nachfolgendem Lungenödem auslösen.

B 70\% aller Lungenemboliepatienten klagen über akuten Husten.

C Plötzlicher Husten und Luftnot sprechen für eine COPD.

D Bei Rhino-, Laryngo- oder Tracheobronchitis finden sich meist Bakterien, selten Viren oder eine Allergie.

E Postinfektiöser Husten klingt unmittelbar nach dem Infekt ab.

\section{- 8. Welche Aussage zu Erkrankungen mit} chronischem Husten trifft zu?
A Asthma geht nur mit Dyspnoe, nicht mit Husten einher.

B Medikamente, z. B. ACE-Hemmer, verursachen nie chronischen Husten.

C Trockener Husten findet sich oft bei diffusen Lungenparenchymerkrankungen.

D Die Gastroskopie ist beweisend für die Diagnose "gastroösophagealer" Reflux.

E Bei Herzinsuffizienz husten die Patienten, wenn sie stehen.

\section{- 9. Ein diagnostischer Leitsatz zum Thema} Husten ist:

A Die genaue Anamneseerhebung ist i. d. R. wegweisend für die Diagnose.

B Bei unklarer Anamnese immer eine Bronchoskopie durchführen.

C Antibiotika gelten als Therapeutikum der ersten Wahl bei Husten.

D Primär vor jeglicher Diagnostik inhalative Noxen und protussive Medikamente vermeiden/absetzen.

E Bei unklarer Ursache sollte man nach spätestens einer Woche einen Röntgen-Thorax durchführen.

\section{- 10. Als therapeutischer Leitsatz gilt:}

A Mukolytika oder Sekretolytika sind in der Langzeittherapie des chronischen Hustens sinnvoll.

B Expektoranzien und Antitussiva nur zeitbefristet einsetzen.

C Ziel der Physiotherapie ist, den produktiven Husten durch optimierte Hustentechnik zu unterbinden.

D Die klinische Wirksamkeit von Expektoranzien ist zweifelsfrei bewiesen.

E Ambroxol, N-Acetylcystein und Myrtol besitzen Evidenzgrad A.

\section{ANTWORTFORMULAR}

Mit Einreichen dieses Fragebogens erkläre ich mich damit einverstanden, dass die angegebenen Daten zum Zweck der Teilnahmebestätigung gespeichert und bei erfolgreicher Teilnahme an den Einheitlichen Informationsverteiler (EIV) der Ärztekammern weitergegeben werden.

Wichtiger Hinweis:

Eine Auswertung ist künftig nur noch möglich, wenn Sie Ihre EFN auf dem Antwortformular angeben! Nähere Hinweise hierzu unter: www.cme-punkt.de/faq.html

\section{„Symptom Husten“}

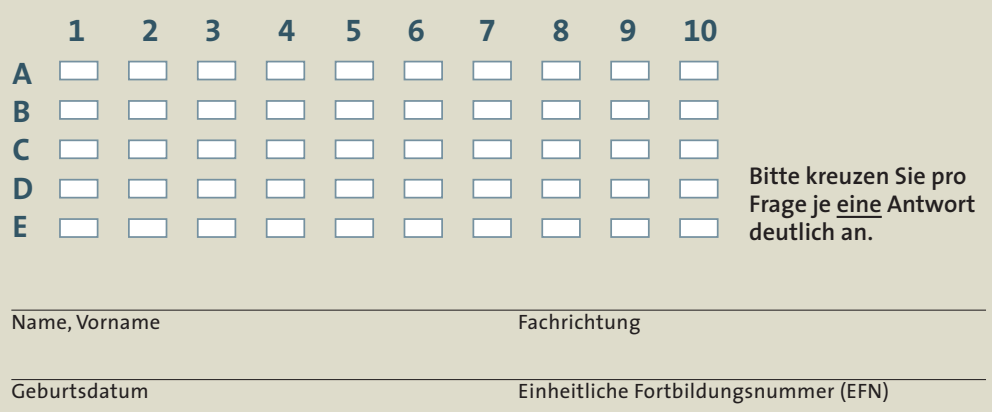

Und so kommen Sie zu Ihren Punkten:

Teilnahme im Internet: unter www.cme-punkt.de. Dort führen wir für Sie ein elektronisches Punktekonto.

Teilnahme per Brief: Fragebogen ausfüllen und mit frankiertem Rückumschlag an: Urban \& Vogel GmbH CME MMW-Fortschr. Med. Postfach 81664 München. 